\title{
Los aportes de la fenomenología de Husserl a la metafísica de Zubiri: una reflexión sobre la conciencia intencional y la impresión*
}

\author{
Alexánder Ávila Martínez**
}

Recepción: 6 de septiembre de 2012 • Aprobación: 11 de diciembre de 2012

El presente texto intenta realizar una mirada al pensamiento de Zubiri, con el propósito de identificar la influencia de la fenomenología de Husserl en el pensamiento zubiriano. Específicamente se trabajan los conceptos de conciencia intencional e impresión. El texto también quiere mostrar la forma en que Zubiri encontró, a partir de la fenomenología, su camino propio para la construcción de su perspectiva metafísica y el análisis de la realidad.

Palabras clave: Husserl, fenomenología, conciencia intencional, impresión, Zubiri.

Artículo de investigación, fruto de la investigación personal que lleva el autor en sus estudios doctorales.

* Licenciado en Filosofía. Magíster en Filosofía. Estudiante de Doctorado en Filosofía de la Universidad Santo Tomás. Líder del grupo de investigación lus-luris de la Universidad Cooperativa de Colombia. Correo electrónico: alexavilam@gmail.com 


\section{The contributions of the phenomenology of Husserl to Zubiri's metaphysics: a reflection on intentional consciousness and impression}

\section{Abstract}

This paper attempts a look Zubiri's thought, in order to identify the influence of Husserl's phenomenology in his thinking. Specifically, we work on the concepts of intentional consciousness and impression. The text also wants to show how Zubiri found, from phenomenology, his own way to build his metaphysical perspective and the analysis of reality.

Keywords: Husserl, phenomenology, intentional consciousness, impression, Zubiri. 
Les apports de la phénoménologie de Husserl à la métaphysique de Zubiri: une réflexion sur la conscience intentionnelle et l'impression

\section{Résumé}

Ce texte veut tourner son regard vers la pensée de Zubiri, dans le but d'identifier l'influence de la phénoménologie de Husserl dans la pensée zubirienne. Plus spécifiquement, sont travaillés les concepts de conscience intentionnelle et impression. Le texte veut aussi montrer la manière que Zubiri a trouvé, en partant de la phénoménologie, son propre chemin pour la construction de sa perspective métaphysique et l'analyse de la réalité.

Mots-clés: Husserl, phénoménologie, conscience intentionnelle, impression, Zubiri. 


\section{Introducción}

La tarea del filósofo es proponer, la tarea del filósofo es indicar, pues se hace más fácil llegar a cualquier parte cuando se tienen indicaciones. Heidegger (1999) menciona: "Mi mentor en la búsqueda fue el Lutero joven; modelo, Aristóteles. Impulsos me los dio Kierkegaard, y los ojos me los puso Husserl" (p. 22). La frase de Heidegger es una alusión directa a la notoria influencia de Husserl en su visión de la filosofía. Xavier Zubiri se mueve en el mismo horizonte, es decir, Zubiri en sus inicios fue fenomenólogo y Husserl le indicó el camino. Para el pensamiento filosófico de Zubiri, la realidad es una formalidad en la impresión. Al hablar de impresión y de conciencia intencional, se ve claramente la influencia fenomenológica de Husserl. Por ello, el presente escrito intenta revisar el diálogo entre Husserl y Zubiri en relación con la conciencia intencional y la percepción, de donde se originará la intuición zubiriana sobre la impresión.

El presente artículo se compone de tres partes que tienen una conexión entre sí: se empieza revisando algunas características de Xavier Zubiri relacionadas con su vida, su obra y su pensamiento. En el ejercicio posterior se realiza una breve reseña sobre la fenomenología y se muestra cómo esta se erigió como una nueva forma de entender el quehacer filosófico que influyó el pensamiento del siglo XX. Este será un momento importante para poder entender las apreciaciones de la tercera parte del presente escrito, en la que se pretende hacer una mirada al pensamiento de Zubiri, para encontrar la forma en que lo influyó Husserl y, además, cómo Zubiri encontró un camino propio para la construcción de su perspectiva metafísica.

\section{Zubiri: un filósofo de vocación}

Zubiri nació en 1898 en San Sebastián, país Vasco, y murió en Madrid en 1983. Sus estudios iniciales fueron en un colegio religioso de la comunidad 
de los maristas. En 1915 ingresó al Seminario de Madrid' como estudiante externo. El mismo año conoció a Ortega, quien lo introdujo en la filosofía de Husserl. Zubiri realizó estudios de filosofía y teología en las universidades de Madrid, Lovaina y Gregoriana. En Lovaina consiguió su licencia en filosofía; en la Gregoriana, su doctorado en teología, y en 1923 se doctoró en filosofía por la Universidad de Madrid, con una tesis dirigida por Ortega: Ensayo de una teoría fenomenológica del juicio.

En 1929 viajó a Friburgo, donde asistió a las últimas lecciones de Husserl y a los primeros seminarios de Heidegger. A mediados de 1930, Zubiri se trasladó a Berlín y estudió ciencias naturales. Estuvo en contacto allí con los creadores de la nueva física: Heisenberg, Schrödinger, Einstein y otros importantes matemáticos y científicos, como Rey Pastor y L. de Broglie. Entre 1936 y 1940 enseñó en la Universidad de Madrid e impartió cursos en París. Luego, entre 1940 y 1942, enseñó en la Universidad de Barcelona. Renunció a la catedra universitaria en 1943, para dedicarse de lleno a la investigación. Su labor como docente se dirigió, desde esa fecha, a la enseñanza en seminarios privados.

\section{Zubiri y su obra}

La obra de Zubiri se muestra sistemática y propone todo un itinerario filosófico. Marquínez (2006) realiza una mirada a la obra de Zubiri en los siguientes términos:

Su primer libro Naturaleza, historia, Dios, en el que se recogía parte de su producción anterior, se publicó en 1944. A finales de 1962 publicó el controvertido tratado Sobre la esencia, y entre 1980-1983 Inteligencia sentiente, su última y definitiva obra. El resto de sus escritos son en su mayoría publicaciones póstumas de los cursos extrauniversitarios (p. 176).

1 Fue ordenado sacerdote, aunque posteriormente, a petición propia, volvió al estado laical para contraer matrimonio con Carmen Castro. 
Otros libros no señalados en la reseña de Marquínez son: Cinco lecciones de filosofía (1963), Inteligencia y logos (1982), Inteligencia y razón (1983), El hombre y Dios (1984), Sobre el hombre (1986), Estructura dinámica de la realidad (1989), Sobre el sentimiento y la volición (1992), El problema filosófico de la historia de las religiones (1993), Los problemas fundamentales de la metafísica occidental (1994), Espacio. Tiempo. Materia (1996), El problema teologal del hombre: cristianismo (1997), El hombre y la verdad (1999), Sobre la realidad (2001).

\section{Zubiri y su pensamiento}

En el pensamiento filosófico de Zubiri confluyen distintas manifestaciones. Suele ubicarse da manera casi esquematizada a la fenomenología de Husserl y a la ontología de Heidegger como los elementos más importantes para la construcción del pensamiento metafísico de Zubiri. Evidentemente, la fenomenología y la ontología ocupan un lugar privilegiado en las intuiciones filosóficas del autor vasco; sin embargo, es preciso mencionar otros elementos que permitieron el desarrollo de la visión filosófica de Zubiri; elementos como el conocimiento claro de lenguas no indoeuropeas y de lenguas clásicas, una vasta formación científica y un conocimiento al detalle de la historia de la filosofía y de la historia de las religiones.

Ahora bien, es el mismo Zubiri (1980), en el prólogo a la traducción inglesa de Naturaleza, historia y Dios, quien establece una etapa previa y dos etapas claras que permitirían la aparición de su pensamiento filosófico. La etapa previa está ligada a la formación fenomenológica anterior al año 1932; la primera etapa, la etapa ontológico-metafísica, está circunscrita entre los años 1932-1944, y la etapa metafísica, entre los años 1944-1983. Otro elemento que se muestra importante cuando se reflexiona sobre Zubiri tiene que ver con su visión de la historia de la filosofía. Zubiri ve a la historia de la filosofía en tres horizontes. En relación con el horizonte, Marquínez (1981) manifiesta lo siguiente: 
Desde M. Kant se viene hablando de un horizonte de nuestro conocimiento, pero especialmente en nuestros días han hecho abundante uso de esta categoría Husserl, Heidegger, Zubiri y Jaspers. El termino horizonte (del gr. Oros, límite) significa en el lenguaje cotidiano aquella línea lejana en la que parece juntarse el cielo con la tierra [...] Ahora bien, dado que nuestro entendimiento es una facultad visiva, podemos hablar de un horizonte de visión intelectual. En este sentido, horizonte es el límite que circunscribe (p. 245).

Para Zubiri, el primer horizonte es el de la movilidad. En este horizonte, los griegos reflexionan acerca del movimiento, y por ello buscan las estructuras de donde se produce el devenir. Para Zubiri, detrás del devenir, detrás del movimiento, se encuentra, para el horizonte de los griegos, el logos. El segundo horizonte es el de la nihilidad, en el que las cosas son pero podrían no ser. Zubiri ubica en este horizonte a la filosofía medieval y a la moderna. San Agustín inicia el horizonte que encuentra su fin con la síntesis de la filosofía occidental elaborada por Hegel. El tercer horizonte se inaugura con el pensamiento de Husserl y de Heidegger; sin embargo, estos autores son una salida insuficiente del tercer horizonte. Por ello, se hace imprescindible ubicar a la metafísica como el tercer horizonte de la filosofía occidental, en el que se elaborará un análisis claro sobre la realidad, que permite ir a la realidad misma de las cosas. Zubiri presenta su visión de la realidad en los siguientes términos:

Recuérdese que me proponía, en primer lugar, enfocar el problema de la realidad. Un enfoque que era averiguar en qué dimensión y en qué forma le es presente al hombre eso que llamamos la realidad. Afirmaba que este enfoque no puede ser sino la índole que uno presupone la inteligencia humana. Esta inteligencia - decía - es una inteligencia sentiente que por modo impresivo me presenta la formalidad de lo real (Zubiri, 2001, p. 235).

A diferencia de Husserl y Heidegger, Zubiri cree que lo que la filosofía estudia no es ni la objetividad ni el ser, sino la realidad en cuanto tal; es 
decir, el elemento central del estudio filosófico tiene que vérselas directamente con la metafísica.

\section{La Fenomenología de Husserl: una reconstrucción de la filosofía}

La obra de Husserl nace en un contexto muy particular. Sus preocupaciones filosóficas iniciales se centraron en la matemática. De hecho, trabajó ampliamente en lo relativo a la filosofía de la aritmética. No obstante, con el advenimiento de la Primera Guerra Mundial, su percepción sobre la tarea de la filosofía cambió, y ello lo llevó a desarrollar dos premisas que se muestran, según Herrera (s. f.), como fundamentales: 1) la eminente correlación entre el hombre y el mundo ${ }^{2}$ y 2 ) la visión del mundo de la vida como verdadero a priori universal que permite la construcción de mundos especializados.

Husserl, al vivenciar de manera directa la Primera Guerra Mundial, creyó inevitable que de la misma forma que era necesario reconstruir el mundo de la posguerra, era necesario también reconstruir a la filosofía. Tal pretensión exigía una transformación del pensamiento, pero sobre todo una transformación de la comprensión de lo que es la naturaleza de la filosofía y, con ello, de su tarea. Husserl (1991), en La crisis de las ciencias europeas y la fenomenología trascendental, muestra cómo "la concepción de la idea de una totalidad de ser infinita sistemáticamente dominada por una ciencia racional es lo radicalmente nuevo" (p. 14).

Es decir, para Husserl, la filosofía que en los inicios del siglo XX venía siendo una mixtura entre positivismo e historicismo y que se apoyaba en la psicología ${ }^{3}$, dejó de tener significado para el hombre; por ende, el

2 Al respecto, Herrera (s. f.) muestra cómo Husserl, en 1898, tuvo la intuición de que entre el hombre y el mundo existe una correlación, es decir, que no se puede comprender al hombre sin su relación con el mundo, ni al mundo sin su relación con el hombre. Según Herrera, la vida académica de Husserl estuvo dominada por la tarea de una elaboración sistemática de este a priori de correlación.

3 Para Husserl, los seres humanos carecemos de un conocimiento absoluto de la verdad; por ello, necesitamos guiarnos por la evidencia: todo conocimiento con pretensión de autenticidad descansa 
propósito de lo nuevo, es decir, de la fenomenología, es enseñar la necesidad de volver sobre las cosas mismas y realizar de estas las descripciones más exhaustivas posibles. Tal perspectiva permite, en primera instancia, evitar la continua recaída sobre puntos comunes $y$, en segundo lugar, enriquecer cada hecho con una multiplicidad de situaciones significativas. Señala Husserl (1982): "Hemos sido llevados hacia las profundidades, y en las profundidades se encuentran las oscuridades y en las oscuridades, los problemas" (p. 101).

La reconstrucción de la filosofía, el salir de las profundidades, está signada a la aparición de la fenomenología. Herrera (s. f.) la define en los siguientes términos: "La fenomenología es un método descriptivo cuyo objetivo es un saber sobre el mundo de la vida, como el verdadero a priori universal concreto de nuestra experiencia, sobre cuyo suelo nos es dado construir muchos mundos especializados (p. 12). Por su parte, Husserl (2006) va a mostrar a la fenomenología como un determinador de los ámbitos científicos. En Meditaciones cartesianas lo muestra así:

La fenomenología tiene que llegar por sí misma a los sistemas de conceptos que determinan el sentido fundamental de todos los ámbitos cientíícos. Estos son los conceptos que predelinean todas las demarcaciones formales de las idea-forma de un posible universo del ser en general $y$, por tanto, también la de un posible mundo en general. De acuerdo con esto, ellos tienen que ser los auténticos conceptos fundamentales de todas las ciencias. (p. 172).

Retomando el proceso de reconstrucción de la filosofía, este requiere de tres actores mínimos: en primer lugar, la refundación del concepto kosmos; en segundo lugar, y en paralelo con el punto anterior, la certeza en la posibilidad de construir una manera de conocer rigurosamente todas las cuestiones significativas, con un método apodícticamente evidente

en la evidencia, y necesariamente hasta donde llega la evidencia llega el conocimiento; sin embargo, la tradición no indagó mucho al respecto, y el trabajo se lo dejó a la psicología, que realizó solo unas pesquisas subjetivas. Por esta razón, Husserl realizó otras pesquisas en las que muestra que cuando se habla de evidencia, se pretende mostrar que es algo que "está dado por sí mismo". 
y en un progreso infinito; en tercer lugar, la convicción de que el lazo que permite atar estos dos extremos inconmensurables se halla en la conmensurabilidad de las formas geométricas puras y de las relaciones matemáticas formales. De esta manera, la reconstrucción de la filosofía le exige al hombre pensar de otra manera, le exige al hombre pensar en primera persona. Husserl (1986) lo afirma así:

Empezamos nuestras meditaciones como hombres de la vida natural, representándonos, juzgando, sintiendo, queriendo en actitud natural. Lo que esto quiere decir nos lo ponemos en claro en sencillas consideraciones, que como mejor las llevamos a cabo es en primera persona (p. 65).

Pensar de otra manera, pensar en primera persona, reconstruir la filosofía implicaría, entonces, tres tareas fundamentales: 1) realizar un conocimiento distinto al conocimiento que realiza la ciencia; 2) realizar un conocimiento mediante una filosofía rigurosa; y 3 ) realizar un conocimiento con un método propio y distinto en relación con las otras ciencias.

\section{El método fenomenológico y la conciencia intencional}

La pretensión de Husserl en relación con el método fenomenológico es demostrar que lo que es real es la vivencia. Por ello, la fenomenología pretende describir y omite explicar; es decir, la fenomenología pretende llegar a las cosas mismas partiendo de la propia subjetividad, en cuanto las cosas se experimentan primariamente como hechos de conciencia. Ahora bien, para Husserl, la visión que se tuvo de la conciencia en la modernidad fue errada, pues se fundamentó en presupuestos ${ }^{4}$; por ello, para Husserl, la conciencia debe entenderse de otra manera, debe entenderse como intencionalidad, como relación directa entre conciencia y objeto.

4 La modernidad, según Husserl, sentó su perspectiva de conciencia como receptáculo en el que se tienen representaciones del mundo. 
Para conseguir esa relación directa entre conciencia y objeto (conciencia intencional), Husserl (1986) mostrará que el "método es visión en el sentido de un aprender a ver, analizar, distinguir y describir" (p. 9). Es decir, en Husserl el método está relacionado con la descripción, y para conseguir las más exhaustivas descripciones se debe llevar a cabo una sucesión de pasos. El primero tiene que ver con la reducción; esta debe ser fenomenológica, eidética y trascendental. En la reducción fenomenológica, según Husserl (1986), la fenomenología pone entre paréntesis la realidad; en la reducción eidética se indaga la esencia del asunto, en tanto que en la reducción trascendental se analiza el mundo como está en la experiencia, es decir, se quita la realidad del mundo y de quien lo describe, para encontrar la pura experiencia de las cosas o epokhé. Después de que se han elaborado las reducciones, se puede llegar a una relación clara entre conciencia intencional y mundo.

\section{Ua reflexión sobre la conciencia intencional y la impresión: el origen de la metafísica de Zubiri}

Zubiri accede a la fenomenología por varias razones. Una de ellas tiene que ver con la notoria influencia de quienes dirigieron sus tesis de licencia y doctorado: L. Noël y Ortega y Gasset; sin embargo, afirmar que Zubiri accedió en primera instancia a la fenomenología de Husserl solo por influencia de sus profesores se muestra problemático, pues aunque estos le mostraron el camino, Zubiri dio sus propios pasos y se centró en la fenomenología objetivista; de esta manera, Zubiri encuentra una plataforma para superar las posturas de la modernidad. Al respecto, Conill (1988) afirma:

\footnotetext{
Zubiri es consciente del fracaso de la modernidad, debido a que el planteamiento "crítico" moderno conduce al "deísmo", término que engloba todas las formas de "filosofía de la conciencia" [...] que se apoyan en ella para acceder a la realidad (p. 219).
} 
La filosofía de Husserl se convierte para Zubiri en la posibilidad más sensata de dejar atrás la filosofía moderna y abrirse a un nuevo plexo de posibilidades en el que se pueda ir a las cosas mismas. Es necesario revisar la modernidad, porque en ella se da primacía a la razón y se deja a la percepción como algo no muy importante.

En la Crítica de la razón pura, Kant (1988) sostiene que el conocimiento no se rige por el objeto, sino el objeto por el conocimiento. En ese orden de ideas, no todo el conocimiento procede de la experiencia. Existe en la primera crítica de Kant una escisión en los juicios: existe una diferencia entre juicios analíticos y juicios sintéticos. Los juicios analíticos aportan mayor claridad, pero no nuevo conocimiento, en tanto que los juicios sintéticos sí generan nuevo conocimiento. Por otro lado, existe también una diferencia notoria entre los juicios a priori y los juicios a posteriori. Los primeros son aquellos que están en el entendimiento y no proceden de la experiencia; los segundos son aquellos que nacen de la experiencia. Para Kant, los juicios más importantes son los juicios sintéticos a priori, pues permiten crear nuevo conocimiento prescindiendo de la experiencia.

De esta manera, para Kant el hombre es, en esencia, su razón. En ese sentido, si el hombre desea vivir como hombre, su comportamiento debe ser racional y debe fundamentarse en el uso puro de la razón, es decir, en la facultad que tiene la razón para establecer razonamientos necesariamente verdaderos al margen de la experiencia. Al respecto, Roberto Aramayo en el estudio preliminar de Crítica de la razón práctica (2009) indica que Kant necesitaba presentar un escrito cuya estructura metodológica guardase una simetría con la primera Crítica, a fin de subrayar que nos las habemos con dos usos (el práctico y el especulativo) de una única razón. Esa única razón mencionada por Aramayo es la razón pura ${ }^{5}$. La razón pura posee dos usos para Kant; y sus obras más reconocidas explicitan sobre dichos usos. La Crítica de la razón pura hace referencia directa al uso de la razón especulativa y a la manera en que esta otorga la posibilidad de un uso teorético, que permite el acceso al conocimiento verdadero de la

5 La razón pura (Vernunft) es utilizada en la Crítica de la razón pura como aquella que contiene los principios a priori del conocimiento. 
naturaleza. La Crítica de la razón práctica hace énfasis en el uso de la razón práctica, y brinda la posibilidad de acceder al conocimiento verdadero sobre los actos humanos.

La obra de Husserl que más influyó al joven Zubiri fue Investigaciones lógicas, específicamente el tema relacionado con la conciencia intencional. Con Investigaciones lógicas Zubiri iniciará su itinerario filosófico, entendiendo que la renovación de la filosofía debe realizarse a partir del estudio fenomenológico de la conciencia y de la reivindicación de la percepción; para Zubiri, la fenomenología se ha encargado de realizar una crítica fuerte a la filosofía moderna, y en especial a la perspectiva que entiende la conciencia como receptáculo en el que se guardan las distintas representaciones que se tienen del mundo. La fenomenología le permitirá entender que la conciencia es siempre darse cuenta de algo, que la conciencia es intuir lo esencial; a partir de Husserl, Zubiri percibirá que lo único que está inmediatamente dado y que constituye el principio de todos los principios no es ni el sujeto ni el objeto, sino la relación de conciencia. Por ello, Zubiri (1980) afirmará que la fenomenología es "el correlato objetivo e ideal de la conciencia" (p. 14).

Para Zubiri, otro elemento significativo de la fenomenología de Husserl tiene que ver con la reivindicación de la percepción. Para Husserl, en la percepción hay un momento intelectivo. Este autor define la verdad como correlativa al acto cognoscitivo, donde el significado encuentra su cumplimiento en la percepción; es decir, la vivencia expresiva y la percepción correspondiente se fusionan en un acto de conocer. La Investigación VI de Husserl se convertirá en la reivindicación de la percepción, y será para Zubiri una salida al horizonte de la nihilidad. Ahora bien, Zubiri deja de ser fenomenólogo cuando entiende que el gran problema de la reducción es que es necesario poner entre paréntesis a la realidad para llegar a la pura experiencia; en ese orden de ideas, la realidad es para Husserl el sentido que se le da a las cosas.

En Zubiri, la realidad no es sentido, sino formalidad. El autor vasco intuye que la filosofía occidental se basó siempre en contenidos, pasando por alto la formalidad, lo cual hizo que la filosofía occidental tuviese una visión 
no adecuada de la realidad. Cuando Zubiri reflexiona sobre la realidad, entiende que esta es alteridad, pues la realidad queda como otra respecto a la impresión misma. Entendiendo así la realidad, esta no remite a mi impresión; por el contrario, la realidad remite al otro. En ese orden de ideas, la impresión de realidad, en la medida en que es impresión, es un acto de sentir; pero en cuanto la impresión es impresión de realidad, Zubiri (2005) muestra la realidad en los siguientes términos:

Ante todo, todo lo real es lo que es solo respectivamente a otras realidades. Nada es real si no es respecto a otras realidades. Lo cual significa que toda cosa real es desde sí misma constitutivamente abierta. Solo entendida desde otras cosas que habrá que buscar, habremos entendido lo que es la cosa que queremos comprender. Lo que así entendemos es lo que la cosa es en la realidad. El arrastre con que nos arrastra la realidad hace, pues, de su intelección un movimiento de búsqueda. Y como esto mismo sucede con aquellas otras cosas desde las que entendemos lo que queremos entender, resulta que al estar arrastrados por la realidad nos encontramos envueltos en un movimiento inacabable no solo porque el hombre no puede agotar la riqueza de la realidad, sino que es inacabable radicalmente, a saber, porque la realidad en cuanto tal es desde sí misma constitutivamente abierta. Es, a mi modo de ver, el fundamento de la célebre frase de San Agustín: "Busquemos como buscan los que aún no han encontrado, y encontremos como encuentran los que aún han de buscar". Investigar lo que algo es en la realidad es faena inacabable, porque lo real mismo nunca está acabado. La realidad es abierta y múltiple (p. 2).

Para Zubiri, la filosofía occidental se quedó en la objetividad y olvidó las impresiones; incluso Husserl se quedó solo en los contenidos, olvidando que la impresión no es igual a lo impreso, que la realidad no es lo que está afuera, sino que la realidad es lo más interno del ser humano, pero a su vez es alteridad. 
Evidentemente, se puede notar un Zubiri que acude a la fenomenología de Husserl, para encontrar una salida a la filosofía de la modernidad, que obvió el papel de la percepción y endiosó a la razón. Husserl también le permite hallar a Zubiri una perspectiva nueva sobre la conciencia, le permite abrir un escenario donde la conciencia debe entenderse de otra manera, debe entenderse como intencionalidad, como relación directa entre conciencia y objeto. Sin embargo, y de manera paradójica, al tiempo en que Husserl le abría los ojos a Zubiri, este ya no era más fenomenólogo; al mismo tiempo, se da una ruptura en la que Zubiri entiende por formalidad lo suyo, es decir, la realidad.

\section{$\mathbf{R}_{\text {eferencias }}$}

Conill, J. (1988). El crepúsculo de la metafísica. Barcelona: Anthropos.

Heidegger, M. (1999). Ontología. Hermenéutica de la faticidad. Madrid: Alianza.

Herrera, D. (s. f.). ¿Qué es la fenomenología? Recuperado http://danielherrerarestrepo.googlepages.com

Husserl, E. (1980). Experiencia y juicio. México: Universidad Nacional Autónoma de México.

Husserl, E. (1982). La idea de la fenomenología. Madrid: Fondo de Cultura Económica.

Husserl, E. (1986). Ideas relativas a una fenomenología pura y una filosofía fenomenológica. México: Fondo de Cultura Económica.

Husserl, E. (1999). Investigaciones lógicas. Madrid: Alianza.

Husserl, E. (1991). La crisis de las ciencias europeas y la fenomenología trascendental. Barcelona: Crítica. 
Husserl, E. (2006). Meditaciones cartesianas. Madrid: Tecnos.

Kant, I. (2009). Crítica de la razón práctica. Madrid: Alianza.

Kant, I. (1988). Crítica de la razón pura. Madrid: Alfaguara.

Marquínez, G. (1981). Horizontes históricos de la metafísica: hacia una metafísica desde Latinoamérica. I Congreso de Filosofía Latinoamericana. Bogotá: Universidad Santo Tomas.

Marquínez, G. (2006). Paul Tillich y Xavier Zubiri: Planteamiento del problema de Dios. Cuadernos de Filosofía Latinoamericana, 95. Bogotá: Universidad Santo Tomás.

Zubiri, X. (1987). Naturaleza, Historia y Dios (9. ${ }^{a}$ ed). Madrid: Alianza.

Zubiri, X. (2001). Sobre la realidad. Madrid: Alianza.

Zubiri, X. (2005). Qué es investigar. The Xavier Zubiri Review, 7. Recuperado de http://www.zubiri.org/general/xzreview/2005/pdf/xzr_2005.pdf 